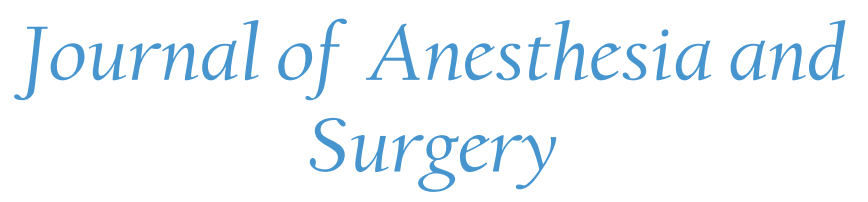

\title{
Proposed Role of Thromboelastometry Assay in Discerning Coagulation Pattern of Patients Suffering Immune-Mediated Thrombocytopenia
}

\author{
Pilar Taura*, Annabel Blasi, $M^{\mathrm{a}}$ Jose Alvira, Ricard Navarro-Ripoll, Lilia Martinez, Juan Carlos Gar- \\ cia-Valdecasas
}

Department of Surgery, Hospital Clinic, Barcelona, Spain

"Corresponding author: Dr. Pilar Taura, Department of Anaesthesiology, Hospital Clinic, University of Barcelona C/ Villarroel 170. 08036 Barcelona, Spain, Tel: +34 9322755 58, E-mail: ptaura7@gmail.com

\begin{abstract}
:
Platelet-derived antigens can trigger immune-mediated responses, the most common of which is heparin-induced thrombocytopenia (HIT). IgG antibodies to platelet factor 4 (PF4)-heparin complexes trigger the clinical manifestations of HIT. Only a subset of these antibodies will activate platelets and release of platelet-derived microparticles (PMPs), which heightened thrombin generation (TG) and fibrin polymerization leading to modify fibrin network structure. All together result in heparin-induced thrombosis/thrombocitopenia (HITT). The effectiveness of HIT laboratory testing by means of immunoassays is largely dependent on the method used whereas functional tests, which can demonstrate the platelet-activating properties of HIT-antibodies are currently limited to a few laboratories. We present 3 patients with suspected diagnosis of HIT. Thromboelastometry $\left(\right.$ ROTEM $\left.^{\circledR}\right)$ assay, with the first derivative of the clot growth velocity curve (V-curve) variables, was used for rapid differential diagnosis. Despite of standard variables similarity, the V-curve differed in the 3 patients. In the patient who developed HITT, the V-curve showed an exceptionally high maximum velocity of fibrin formation $\left(22 \mathrm{~mm} \mathrm{~s}^{-1}\right)$ with a critically low maximal lysis $(1 \%)$, suggesting procoagulant state, whereas these values were normal $\left(10 \mathrm{~mm} \mathrm{~s}^{-1}\right.$ and $12 \%$ respectively) in the patient without HITT. In the patient with a definitive diagnosis of tirofiban-induced severe thrombocitopenia, maximum velocity of fibrin formation was markedly low $(5 \mathrm{~mm}$ $\mathrm{s}^{-1}$ ), with moderate hyperfibrinolysis $(21 \%)$ indicating hypocoagulable state. In patients suffering from immune-mediated thrombocytopenia viscoelastic assays could provide useful information.
\end{abstract}

Received date: February 10, 2017

Accepted date: March 3, 2017

Published date: March 10, 2017

Citation: Taura., P., et al. Proposed Role of Thromboelastometry Assay in Discerning Coagulation Pattern of Patients Suffering Immune-Mediated Thrombocytopenia. (2017) J Anesth Surg 4(1): 23-26.

DOI: $10.15436 / 2377-1364.17 .066$

Keywords: Clot strength, Fibrinolysis, Thromboelastometry, Thrombocytopenia, Thrombosis risk

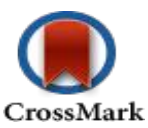

\section{Introduction}

Accurate differential diagnosis between heparin-induced thrombocytopenia with or without thrombosis (HITT and HIT, respectively) and other drug-induced thrombocytopenia (DITP) is needed to reduce the risk of serious thrombotic events or to avoid unnecessary discontinuation of heparin. HIT, the most frequent and serious adverse effect of heparin, often in the setting of inflammation, is caused by auto antibodies against platelet factor 4 (PF4-heparin complexes) which cross-link to platelet Fc $\gamma$ RIIa receptors, leading to platelet activation and release of platelet-derived microparticles (PMPs). Microparticles derived from a variety of cell types, including platelets, mono- cytes and endothelial cells, are strongly procoagulant because they enhance thrombin generation (TG) with profound effects on the final network structure of fibrin $\operatorname{clots}^{[1,2]}$ with a tightly knit network of thin fibers that are resistant to enzymatic lysis ${ }^{[3]}$. However, only a subset of patients whose antibodies evidence strong platelet-activating properties develop HITT. Therefore, the development of a prothrombotic state in the setting of a dropping platelet count is a relatively unique aspect of HITT that distinguishes it from HIT ${ }^{[4]}$. Differential diagnosis through a combination of clinical observations ${ }^{[5]}$ (the $4 \mathrm{~T}$ scoring system) and immune-and-functional assays to detect antibodies and platelet activation ${ }^{[6,7]}$, and $\mathrm{TG}$ assays ${ }^{[8]}$, is time-consuming and/ or require technical expertise. A study of 3 cases is presented an- 
alyzed whether dynamic clot fibrin formation by rotation thromboelastometry (ROTEM ${ }^{\circledR}$, Pentapharm, Munich, Germany) can discern the coagulation pattern of patients with immune-mediated thrombocytopenia.

\section{Case Reports}

After patients consent, approval for publication was obtained from the ethics committee of the Hospital Clinic of Barcelona.

Case 1: A 70-year-old female was admitted to the emergency service with a diagnosis of endocarditis due to pacemaker-lead infection. On admission, the medical treatment was intravenous antibiotics and antiaggregants [oral rivaroxaban and acetylsalicylic acid (ASA)]. Echocardiography showed severe patent foramen ovale (PFO) and moderate tricuspid insufficiency without pulmonary hypertension. Antiaggregants were withdrawn and unfractionated heparin (UFH) was started for thrombosis prophylaxis. Five days later, the patient showed diplopia, and computed tomography revealed embolic stroke of the left superior cerebellar artery ${ }^{[9]}$. The symptoms improved slowly, but 3 days later her condition deteriorated rapidly. She was lethargic and hemodynamically unstable, with oxygen saturations below $90 \%$, and was transferred to the ICU. The platelet count had decreased $>50 \%$ (from 284 to $110 \times 10^{9} \mathrm{~L}^{-1}$ ), but the clinicians considered it to be sepsis-related. Over the next day, platelet count decreased to a nadir value of $12.2 \times 10^{9} \mathrm{~L}^{-1}$ and HITT was suspected, with a high pretest probability (a 4T score of 7 points). UFH was withdrawn and intravenous infusion of argatroban (Grade 1C recommendation $)^{[10]}$ was started with initial doses of $2 \mu \mathrm{g} / \mathrm{k} / \mathrm{min}$ and subsequent doses adjusted to a 2.5-to 3.0-fold prolongation of aPTT. The enzyme-linked immuno-sorbent assay (ELISA) for IgG antibodies against PF4/heparin and ROTEM (see below) were performed. Computer tomography pulmonary angiography suggested pulmonary embolism. The ELISA specific IgG test was positive ( $\mathrm{PF} 4$-heparin $\mathrm{OD} \geq 0.45$ ). A gradual hemodynamic improvement was observed, and 2 days later platelet count was increasing progressively $\left(29 \times 10^{9} \mathrm{~L}^{-1}\right)$. Ten days later, platelet count increased to $120 \times 10^{9} \mathrm{~L}^{-1}$ and it was decided to proceed with surgery (to close the PFO and remove the pacemaker). Argatroban infusion was maintained during surgery and used as an antithrombotic strategy in cardiopulmonary bypass. On the fourth postoperative day (POD) argatroban was withdrawn and oral rivaroxaban was started. There were no additional thromboembolic events or bleeding complications. The subsequent course in the ICU and afterwards was unremarkable and she was discharged to rehabilitation 17 days after surgery.

Case 2: A 59-year-old female underwent an elective hepaticojejunostomy for choledochal cyst. Her medical history was unremarkable. Thromboprophylaxis was started 12 hours before surgery by low- molecular weight heparin, enoxaparin $80 \mathrm{mg}$ (1 $\mathrm{mg} / \mathrm{k}$ ) once daily. The intraoperative and postoperative course behavior was completely usual, but on the five POD thrombocytopenia was evidenced, with a platelet count decrease from 268 to $11.9 \times 10^{9} \mathrm{~L}^{-1}$. HIT was suspected with an intermediate pretest probability (a 4T score of 5 points). Enoxaparin was withdrawn and $5 \mathrm{mg}$ once daily of subcutaneous fondaparinux (Grade 2C), was started. Antibodies were determined and ROTEM was per- formed (see below). The ELISA IgG test was positive (OD $\geq$ 0.45 ). Platelet count began to increase 30 hours after; and on the ninth POD it was over $150 \times 10^{9} \mathrm{~L}^{-1}$. Fondaparinux was withdrawn and 4 days later she was discharged with oral anticoagulation. No thrombotic events were observed. After the follow-up period of 3 months, the anticoagulation prophylaxis was withdrawn.

Case 3: A 55-year-old female with ductal adenocarcinoma was scheduled to undergo elective pancreaticoduodenectomy. The medical history indicated acute myocardial infarction 4 months before treated with 2 pharma-active stents and anti-aggregant with ticagrelor $90 \mathrm{mg}$ plus ASA $100 \mathrm{mg}$. Seeing the high thrombotic risk it was decide to initiate thrombotic prophylactic treatment with intravenous tirofiban $(0.1 \mu \mathrm{g} / \mathrm{k} / \mathrm{min})$ one week before surgery. Tirofiban was stopped 12 hours before surgery and subcutaneous enoxaparin $40 \mathrm{mg}$ twice daily was initiated. Surgery proceeded uneventfully. In the ICU tirofiban was reintroduced. Twelve hours later she developed severe thrombocytopenia with a nadir value of $5.9 \times 10^{9} \mathrm{~L}^{-1}$ and HIT was initially suspected. Specific ELISA and ROTEM were performed. The low pretest of 3 points together with platelet count $<10 \times 10^{9}$ $\mathrm{L}^{-1}$ and ROTEM profile resembling hypocoagulable state (see below) a tirofiban-induced-thrombocytopenia was suspected, and the drug was withdrawn ${ }^{[11]}$. Owing to the potential bleeding risk, platelets were transfused with a limited result $\left(12 \times 10^{9} \mathrm{~L}^{-1}\right.$ increase). As expected, specific ELISA test was negative. Two days later, with a platelet count of $46.2 \times 10^{9} \mathrm{~L}^{-1}$, ticagrelor and ASA were reinstated. The platelet count increased progressively, reaching $148 \times 10^{9} \mathrm{~L}^{-1}$ on the seventh POD. The medical course was unremarkable and she was discharged on the $13^{\text {th }}$ POD and counselled to avoid future exposure to the drug.

\section{Rotation thromboelastometry whole blood analysis}

Standard ROTEM assay was performed in triplicate and handled in the same manner: the blood sample $(2.7 \mathrm{ml})$ was transferred to a polypropylene tube containing $0.5 \mathrm{ml}$ of $3.2 \%$ sodium citrate, and the sample was kept pre-warmed $\left(37^{\circ} \mathrm{C}\right)$ during $45 \mathrm{~min}$. Assessment of kinetics of whole blood clot formation was based on standard and derived parameters of the first derivative curve (V-curve) in ex-TEM test: clotting time (CT, s); clot formation time (CFT, s); maximum clot firmness or clot strength $\left(\mathrm{MCF}_{\text {EXTEM }}, \mathrm{mm}\right.$ ); maximum clot fibrin formation (MaxVel, mm $\mathrm{s}^{-1}$ ); time to reach maximal clot fibrin formation ( $\mathrm{t}-\mathrm{MaxVel}, \mathrm{s}$ ), and velocity (acceleration of the propagation phase of fibrin formation) calculated by the formula MaxVel/t-MaxVel ratio (mV/ $\mathrm{t}-\mathrm{m}, \mathrm{mm} \mathrm{s}^{-2}$ ). Clot lysis at 60 minutes (LI60), and maximum clot lysis (ML\%) were also recorded.

Analysis of ROTEN data: In all patients, we observed a highly reduced $\mathrm{MCF}_{\text {EXTEM }}$ as a consequence of severe platelet count decrease (Figure 1-A). In case 1, ROTEM was characterized by an exceptionally high MaxVel and velocity with a critically low maximum lysis (Figure 1-B and Table 1). In case 2 (Table 1), all parameters analyzed were normal. In case 3, ex-TEM assay showed prolonged CFT, highly reduced MaxVel and velocity and increased maximum lysis (Figure 1-B and Table 1). 

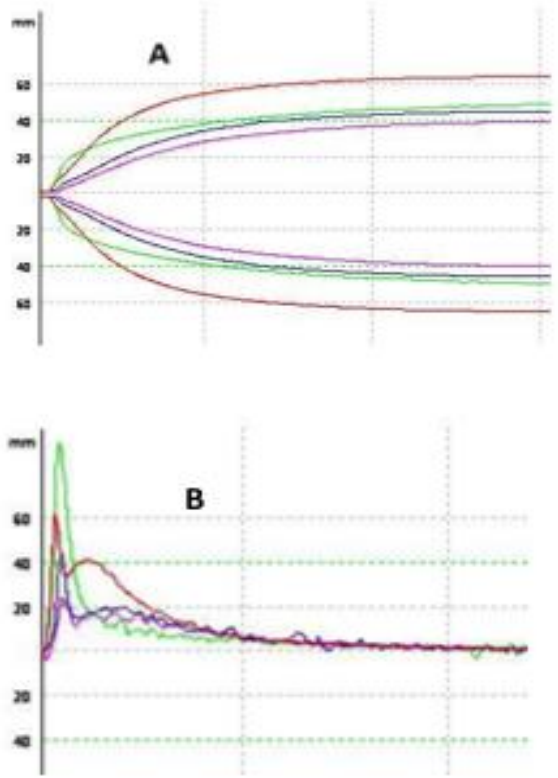

Figure 1: ROTEM standard and derived tracings of the 3 patients suffering immune-induce thrombocytopenia. Top: (A) ex-TEM tracing. Bottom: (B) V-curve kinetic of the 3 reported patients. Patient with HITT (green), patient with HIT (blue), patient with DITP (purple), and healthy adult female (red).

Table 1: Kinetics of the clot growth generated by whole blood clotting rotation thromboelastometry: initiation: (CT and $\mathrm{CFT}$ ); propagation: (MaxVel and t-MaxVel), firmness (MCF); and lysis (LI60 and ML) Platelet count and fibrinogen at the time of thromboelastography of the three patients and healthy adult female.

\begin{tabular}{|c|c|c|c|c|}
\hline & Case 1 & Case 2 & Case 3 & Healthy female \\
\hline CT (s) & 63 & 64 & 71 & 59 \\
\hline CFT (s) & 92 & 107 & 288 & 105 \\
\hline $\operatorname{MaxVel}\left(\mathrm{mm} \mathrm{s}^{-1}\right)$ & 22 & 10 & 5 & 13 \\
\hline t-MaxVel (s) & 83 & 79 & 82 & 74 \\
\hline $\mathrm{mV} / \mathrm{t}-\mathrm{m}\left(\mathrm{mm} \mathrm{s}^{-2}\right)$ & 2.6 & 1.2 & 0.6 & 1.7 \\
\hline MCF ex-TEM (mm) & 47 & 45 & 41 & 65 \\
\hline LI60 & 0 & 8 & 16 & 4 \\
\hline ML (\%) & 1 & 12 & 21 & 9 \\
\hline Nadir platelet count/L & 12.200 & 11.900 & 5.900 & 208.421 \\
\hline Fibrinogen (g/l) & 4.9 & 4.1 & 4.6 & 1.9 \\
\hline
\end{tabular}

Definition of abbreviations: CT: clotting time; CFT: clot formation time; MaxVel: maximum velocity; t-MaxVel: time to reach maximum velocity; MCF: maximum clot firmness; LI60: lysis at 60 minutes of MCF; ML: maximum lysis.

\section{Discussion}

Patients with heparin-induced thrombocytopenia are known to be hypercoagulable, and susceptible to both thrombocytopenia and thrombosis, precipitated, at least in part, by platelets that have become activated via their interaction with autoimmune antibody/antigen complexes. Platelet factor 4, PF4, is synthesized in megakaryocytes, packaged into platelet $\alpha$-granules, and released upon platelet activation binds to circulating heparin in patients treated with unfractionated or low molecular weight heparin in the vascular endothelial wall. Therapeutic con- centrations of heparin disrupt antibody binding in part by eluting PF4 from the platelet surface, which reduces formation of antigenic complexes. Variation in the expression of platelet-derived PF4 might help to explain why only a small percentage of patients who generate antibodies to PF4/heparin develop HIT. Sub threshold levels of antibodies and also non-platelet-activating antibodies (example IgA, IgM) make platelet activation unlikely and so the PMPs, that are responsible for strengthening procoagulant activity, cannot be generated. PMPs increase rates of TG and fibrin formation, with profound effects on the fibrin architecture. They cause the formation of a tightly knit network of thin fibers that are resistant to enzymatic lysis ${ }^{[3]}$. A recent study showed that PF4-heparin antibody complex induced monocyte tissue factor (TF) expression and MPs release suggesting that monocyte-derived TF- MPs rather than platelet-derived MPs initiate and aggravate thrombosis in HIT ${ }^{[12]}$.

The effectiveness of HIT laboratory testing by means of immunoassays is largely dependent on the method used whereas functional tests, which can demonstrate the platelet-activating properties of HIT-antibodies, may accurately diagnose or rule out HIT, but they are currently limited to a few laboratories. The head bedside whole-blood viscoelastic assays provide information of fibrin polymerization under oscillatory low-shear. The principal measurement is MCF which reflects the clot firmness stabilized by interaction of platelets with polymerized fibrin, and factor XIII. Since the platelet contractile forces are the major contributor to overall clot strength, very low MCF values can be expected in patients with thrombocytopenia. However, in patients with severe immune thrombocytopenia we observed nearly normal values of MCF (range 49 to $71 \mathrm{~mm}$ ), regardless of the diagnosis. The possible mechanistic explanation is that large amounts of fibrinogen, habitually HIT appear in the context of inflammation (e.g. surgical postoperative period, intensive care patients, trauma...), are captured by a few activated platelets via the abundant GPIIb/IIIa receptors on the surface of a platelet $(\sim 50.000 \text { per platelet })^{[13,14]}$ even in the presence of a platelet count $<10 \times 10^{9} \mathrm{~L}^{-1}$ and thus, clot strength increases in a fibrinogen concentration-dependent manner. As a result, standard thromboelastographic parameters are unusable to detect thrombotic risk related to immune-mediated thrombocytopenia. However, the pattern produced from the first derivative, the V-curve, provides information about fibrin clot kinetics (the speed and intensity of fibrin polymerization) displaying a considerable degree of "profile similarity" to the "HIT thrombogram profile" described by Tardy-Poncet and coworkers, and obtained by calibrated automated thrombinography ${ }^{[7]}$. These authors demonstrate that, paradoxically, in HIT-positive patients the presence of heparin enhances the velocity and the peak of TG ( MaxVel), whereas the opposite occurs in HITT-negative patients.

In our patient with HIT, normal ROTEM parameters indicated nonappearance of thrombotic risk and no reason to stop heparin. However, at present, fibrin polymerization kinetics offers no reliable support to taking clinical decisions in this setting. In the context of DITP, thromboelastometry may offer additional information about bleeding risk until platelet count recovery, and may also help determine the need for platelet transfusion.

Our findings suggest that the thromboelastometry, specifically the pattern produced from the first derivative of velocity waveform, V-curve, deserves further investigation as an 
easy and inexpensive bedside tool that may help in monitoring patients confirmed or suspected immune-mediated thrombocytopenia.

Conflict of interests: The authors state that they have no conflict of interest.

\section{References}

1. Warkentin, T.E. HITlights: a career perspective on heparin-induced thrombocytopenia. (2012) Am J Hematol Suppl 1: S92-99.

Pubmed I Crossref I Others

2. Hughes, M., Hayward, C.M., Warkentin, T.E., et al. Morphological analysis of microparticle generation in heparin-induced thrombocytopenia. (2000) Blood 96(1): 188-194.

Pubmed I Others

3. Zubairova, L.D., Nabiullina, R.M., Nagaswami, C., et al. Circulating microparticles alter formation, structure, and properties of fibrin clots (2015) Sci Rep 5: 17611

Pubmed I Crossref I Others

4. Greinacher, A., Farner, B., Kroll, H., et al. Clinical features of heparin-induced thrombocytopenia including risk factors for thrombosis: a retrospective analysis of 408 patients. (2005) Thromb Haemost 94(1): 132-135.

Pubmed I Crossref I Others

5. Lo, G.K., Juhl, D., Warkentin, T.E., et al. Evaluation of pretest clinical score (4 T's) for the diagnosis of heparin-induced thrombocytopenia in two clinical settings. (2006) J Thromb Haemost 4(4): 759-765.

Pubmed I Crossref I Others

6. Cuker, A. Clinical and laboratory diagnosis of heparin-induced thrombocytopenia: an integrated approach. (2014) Semin Thromb Hemost 40(1): 106-114.

Pubmed I Others
7. Malicev, E., Kozak, M., Rozman, P. Evaluation of a flow cytometric assay for the confirmation of heparin-induced thrombocytopenia. (2016) Int J Lab Hematol 38(3): 240-245.

Pubmed I Crossref I Others

8. Tardy-Poncet, B., Piot, M., Chapelle, C., et al. Thrombin generation and heparin-induced thrombocytopenia. (2009) J Thromb Haemost 7(9): 1474-1481.

Pubmed I Crossref I Others

9. Warkentin, T.E., Bernstein, R.A. Delayed-onset heparin-induced thrombocytopenia and cerebral thrombosis after a single administration of unfractionated heparin. (2003) N Engl J Med 348(11): 1067-1069. Pubmed I Crossref I Others

10. Warkentin, T.E., Greinacher, A., Koster, A., et al. Treatment and prevention of heparin-induced thrombocytopenia: American College of Chest Physicians Evidence-Based Clinical Practice Guidelines (8th Edition). (2008) Chest 133(6 Suppl): 340S-380S

Pubmed

11. Bougie, D.W., Wilker, P.R., Wuitschick, E.D., et al. Acute thrombocytopenia after treatment with tirofiban or eptifibatide is associated with antibodies specific for ligand-occupied GPIIb/IIIa. (2002) Blood 100(6): 2071-2076.

Pubmed I Others

12. Rauova, L., Hirsch, J.D., Greene, T.K., et al. Monocyte-bound PF4 in the pathogenesis of heparin-induced thrombocytopenia. (2010) Blood 116(23): 5021-5031.

Pubmed I Crossref I Others

13. Wagner, C.L., Mascelli, M.A., Neblock, D.S, et al. Analysis of GPIIb/IIIa receptor number by quantification of 7E3 binding to human platelets. (1996) Blood 88(3): 907-914. Pubmed I Others

14. Lang, T., Toller, W., Gütl, M., et al. Different effects of abciximab and cytochalasin D on clot strength in thromboelastography. (2004) J Thromb Haemost 2(1): 147-153.

Pubmed I Crossref I Others
Ommega Online Publishers

Journal Title: Journal of Anesthesia and Surgery (JAS)

Journal Short Name: J Anesth Surg
Journal ISSN: 2377-1364

E-mail: anestheisa@ommegaonline.com

Website: www.ommegaonline.org 